\title{
Research Article \\ Flexible Microwave Tag System Based on DGS Multiple Resonators
}

\author{
Seok-Jae Lee, ${ }^{1}$ Jongsik Lim, ${ }^{2}$ Dal Ahn, ${ }^{2}$ and Sang-Min Han ${ }^{1}$ \\ ${ }^{1}$ Department of Information and Communication Engineering, Soonchunhyang University, Asan, \\ Chungnam 336-745, Republic of Korea \\ ${ }^{2}$ Department of Electrical Engineering, Soonchunhyang University, Asan, Chungnam 336-745, Republic of Korea
}

Correspondence should be addressed to Sang-Min Han; auspice@ieee.org

Received 2 April 2013; Accepted 13 May 2013

Academic Editor: Haiwen Liu

Copyright (C) 2013 Seok-Jae Lee et al. This is an open access article distributed under the Creative Commons Attribution License, which permits unrestricted use, distribution, and reproduction in any medium, provided the original work is properly cited.

\begin{abstract}
A new flexible microwave tag system using a frequency-scanning type RFID is proposed with multiple resonators based on defected ground structures (DGSs). The proposed system achieves fully passive tag systems using multiple resonators with a spiral-shaped DGS over a wide frequency range. The resonator implemented on the rear side of a transmission line has the advantages of excellent band notch characteristics as well as bit-error avoidance from the frequency selective reflection. In addition, the tag system is designed on a thin flexible substrate in order to be applicable for amorphous surfaces. The proposed microwave tags have been implemented with wideband antennas at 3-7 GHz on thick and thin flexible substrates. The flexibility of the thin substrate has been evaluated in terms of cognitive capability for various radiuses of curvatures. From the experimental results in an anechoic chamber, the excellent recognition of various multibits identification codes in a wireless transmission environment has been verified.
\end{abstract}

\section{Introduction}

Since the sensor network was issued with various short-range wireless connectivity technologies, interest has been shown in diverse applications for the recognition of identifications (IDs) that can replace bar code systems. Conventional ID systems have developed from a contact type RFID at $13.56 \mathrm{MHz}$ to a contactless type at a UHF band to increase code capacity. Because the recent RFID is used in various ways in collaboration with mobile communication networks, it needs to be of low cost, low power, and large data capacity. The near field communication (NFC) and the chipless RFID become the promising technology candidates for next generation ID systems [1-3].

As the chipless RFID technology has pursued the fully passive tag without semiconductor chips, the ID code should be represented by another feature such as a signal response $[4,5]$, phase/delay difference $[6,7]$, or spectral signature $[6,8-10]$, that is, realized through electromagnetic resonances. However, the conventional chipless RFID has been implemented on a hard plate to maintain the shape of the electromagnetic resonator. Moreover, the resonant circuits shown on the same surface as the receiving and transmitting antennas make a direct reflection of an interrogating signal, which affects the spectral characteristics of the decoded signals. The flexible fabrication of the microwave resonators on the flexible PCBs [11] and textiles [12] has been issued for patch antennas as part of the system components. However, because the tag system should be fully integrated with all circuit components, it is required to be designed on a flexible media which is able to attach to amorphous shaped items.

In this paper, a fully integrated tag system is designed for all passive components on a planar flexible thin substrate. The system can be fabricated using a one-step printing technology to achieve an inexpensive tag price, while the bendable circuit on the flexible substrate can make it feasible for more various applications of the identifications. Moreover, the architecture of the tag is considered for isolated resonance and high resolution. The multiple resonator circuit with a defected ground structure (DGS) is designed with a spiral shape for 


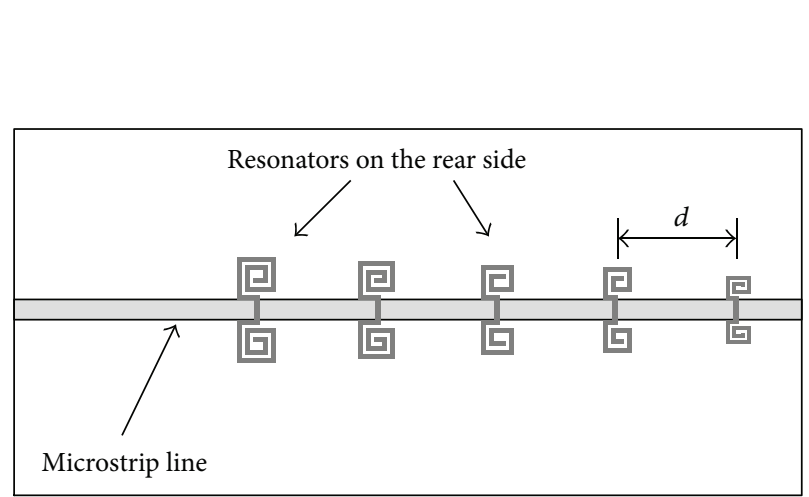

(a)

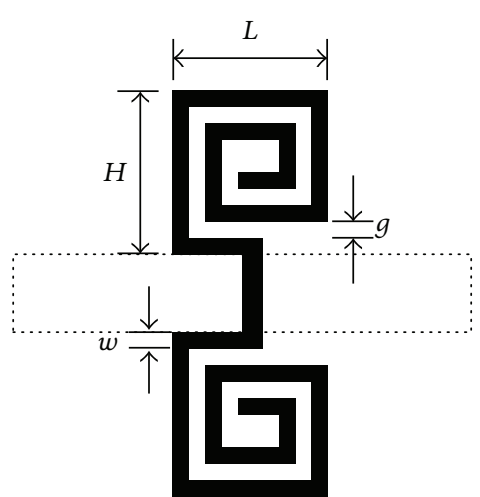

(b)

FIGURE 1: Layout of the multiple resonator circuit using defected ground structures. (a) Multiple resonator circuits. (b) Spiral-shaped DGS resonator.

band notch characteristics of a high Q-factor $[13,14]$. In addition, thanks to the etched ground structures on the back sides of PCBs, the architecture can avoid direct reflection phenomena. Therefore, the microwave interrogating system can reduce the effect of interferences and achieve a long cognitive distance compared with conventional RFIDs.

This paper is organized as follows. Section 2 introduces the design of the flexible microwave tag systems on thick and thin substrates. The system implementation is described in Section 3. Section 4 shows the experimental results of the system performance evaluations. Finally, the commercial feasibility of the proposed system is mentioned in Section 5.

\section{Design of a Flexible Tag with Multiple DGSs}

In frequency scanning type chipless tag systems, an interrogator exposes frequency-swept microwave signals with constant amplitude and linear phase onto the tag surfaces. The received frequency components experience the series multiple resonant characteristics of the amplitude depth and phase nonlinearity at the assigned resonant frequencies. These variations can be recognized from a retransmitted wideband signal at a reader system. In order to detect the resonant bits clearly, the reader needs to receive the only resonant characteristics from the tag. As the multiresonatorbased chipless tag has several resonators located on the same surface with a receiving antenna $[6,8,9]$, the wideband $\mathrm{RF}$ is reflected by the resonant circuits that operate as a frequency selective surface (FSS), producing a noisy interferer that prevents fine decoding at a reader. Therefore, these types of tag systems should be designed with consideration of electromagnetic reflections.

In this section, multiple resonator circuits with DGSs and planar wideband antennas are designed for the microwave tag systems. Figure 1(a) presents the layout of the proposed multiple resonator circuit. The resonator is designed with a DGS on the rear side of a substrate, while a transmission line is solely implemented on the front side on which the wideband antenna is mounted. The separation distances between each resonator are assigned for the isolation and resolution, which are determined as $d=12 \mathrm{~mm}$ and $15 \mathrm{~mm}$ for thick and thin substrates, respectively. The DGSs are designed as a spiral shape for the band notch characteristic with excellent Q-factor and can be directly mounted on the rear side of a microstrip line. The notch represents a digital bit of "ONE," whereas the code "ZERO" is recognized by the frequency component without the notch. Therefore, the code can be designed with the presence of resonators.

The multiple resonators are designed on two types of substrates: a thick substrate for design feasibility and a thin substrate for fabrication flexibility at 3-7 GHz. Figure 1(b) shows the spiral DGS layout. By considering the circuit size and notch performance, the DGSs are designed as dual spirals with a maximum of two windings. Each arm has a symmetric square of height $H$ and length $L$. The line width $w$ and the gap size $g$ are adjusted to control resonant frequencies and $\mathrm{Q}$-factors and are determined up to the fabrication limit to achieve a Q-factor that is as high as possible.

For the implementation on the thick substrate, five types of spiral-shaped DGSs have been designed for 5 bits with more than $10 \mathrm{~dB}$ resolution at each center frequency $\left(f_{o}\right)$ of $3 \mathrm{GHz}, 3.45 \mathrm{GHz}, 4.25 \mathrm{GHz}, 5.2 \mathrm{GHz}$, and $6.2 \mathrm{GHz}$, respectively. The thick substrate employs FR4 with a thickness of $1 \mathrm{~mm}$ and a dielectric constant of 4.4. The design performances have been investigated using a commercial software, the high frequency structure simulator (HFSS) of the ANSYS lnc. Figure 2 presents the design results of the multiple DGS resonators with code "11111." Each DGS operates to a well-matched performance with $-30 \mathrm{~dB}$ to $-40 \mathrm{~dB}$ resonant characteristics and more than $10 \mathrm{~dB}$ resolution.

Flexible multiple resonators are designed on a thin substrate of the RT/Duroid 5880 with a thickness of 5 miles and a dielectric constant of 2.2. Four bits' codes are realized with resonant frequencies of $2.95 \mathrm{GHz}, 3.45 \mathrm{GHz}, 4.2 \mathrm{GHz}$, and $5.55 \mathrm{GHz}$, respectively. Three types of codes are investigated using the same method as that used on the thick substrate. The design results are compared for various codes, as shown in Figure 3. Each bit is evaluated to be clearly recognized 


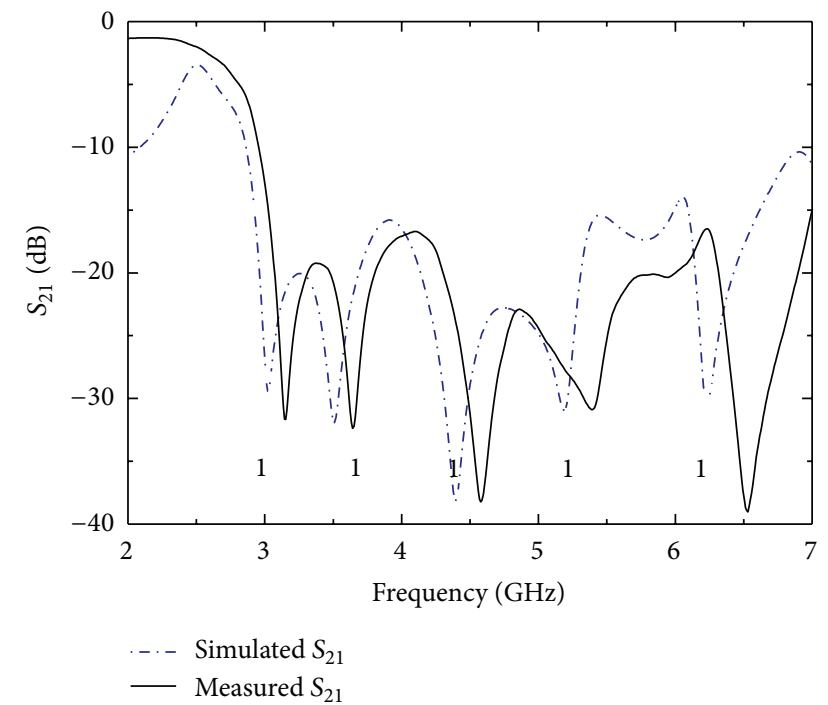

FIGURE 2: Simulated and measured results of multiple resonators on the thick substrate.

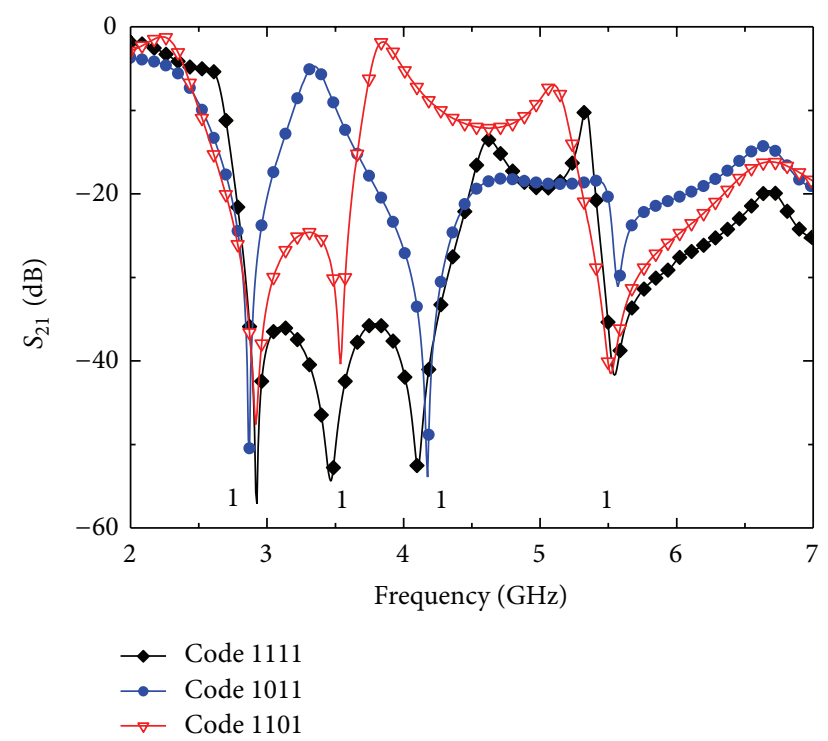

FIGURE 3: Simulated results for frequency resonant bits on the flexible substrate.

by differentiating amplitudes. Table 1 summarizes the design parameters of the multiple resonators on thick and thin substrates.

The tag antenna is designed at an ultrawideband of 2$8 \mathrm{GHz}$ to cover the all resonant frequencies of total resonant bits at $3-7 \mathrm{GHz}$. It is designed as a planar monopole type to be integrated on the same substrate as the multiple resonator circuit [15-17]. Figure 4 presents the layout of the tag antenna. The planar antenna consists of a radiation patch and a feedline on a substrate with an etched ground plane. The feedline $W_{f} \times L_{f}$ is implemented on a microstrip line with and without a ground plane. The resonant frequency is determined by

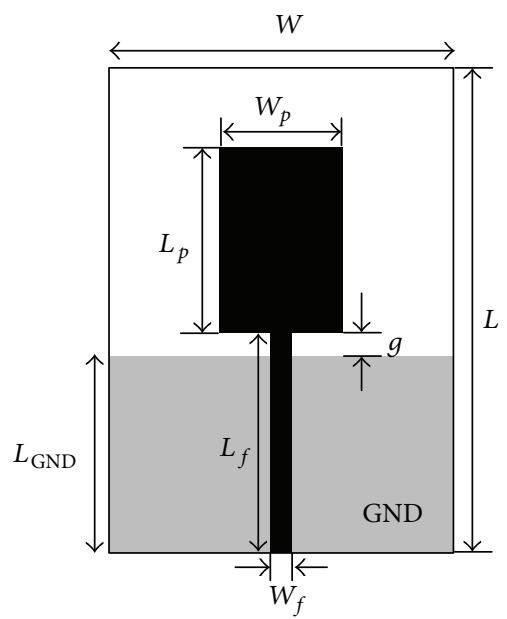

Figure 4: Wideband monopole antenna layout.

TABLE 1: Design parameters of the multiple resonators (dimension: $\mathrm{mm})$.

\begin{tabular}{cccccc}
\hline & $f_{o}(\mathrm{GHz})$ & $H$ & $L$ & $w$ & $g$ \\
\hline \multirow{4}{*}{ Thick substrate } & 3.0 & 4.0 & 3.8 & 0.4 & 0.4 \\
& 3.45 & 3.6 & 3.6 & 0.4 & 0.4 \\
& 4.25 & 3.2 & 3.2 & 0.4 & 0.4 \\
& 5.2 & 3.0 & 2.8 & 0.4 & 0.4 \\
Thin substrate & 6.2 & 2.2 & 2.4 & 0.3 & 0.3 \\
\hline & 3.45 & 4.2 & 4.2 & 0.3 & 0.3 \\
& 4.2 & 4.4 & 4.4 & 0.4 & 0.4 \\
& 5.55 & 3.0 & 3.0 & 0.3 & 0.3 \\
\hline
\end{tabular}

TABLE 2: Design parameters of the wideband antennas.

\begin{tabular}{lcccccccc}
\hline & $W$ & $L$ & $W_{P}$ & $L_{P}$ & $g$ & $W_{f}$ & $L_{f}$ & $L_{\mathrm{GND}}$ \\
\hline Thick & 31.2 & 44.2 & 14.0 & 17.0 & 2.0 & 1.9 & 20.2 & 18.2 \\
Thin & 33.5 & 46.7 & 12.3 & 16.5 & 1.0 & 0.4 & 20.2 & 19.2 \\
\hline
\end{tabular}

the monopole patch design $W_{p} \times L_{p}$. The ground size $W \times$ $L_{\mathrm{GND}}$ and the gap $g$ between the antenna and the ground are considered as the design parameters for a wide bandwidth. The boundary of the ground plane becomes an important matching parameter as well as a ground reflector. Table 2 presents the design results for both substrates.

As shown in Figure 5, the design and experimental results cover the frequency band of tag systems. The wideband antenna on a thick substrate presents the bandwidth of $2.6 \mathrm{GHz}$ to $8.0 \mathrm{GHz}$, while that on a thin substrate shows $2.7 \mathrm{GHz}$ to $8.2 \mathrm{GHz}$. The specification of the antenna is satisfied with both the Tx. and Rx. tag antennas. 


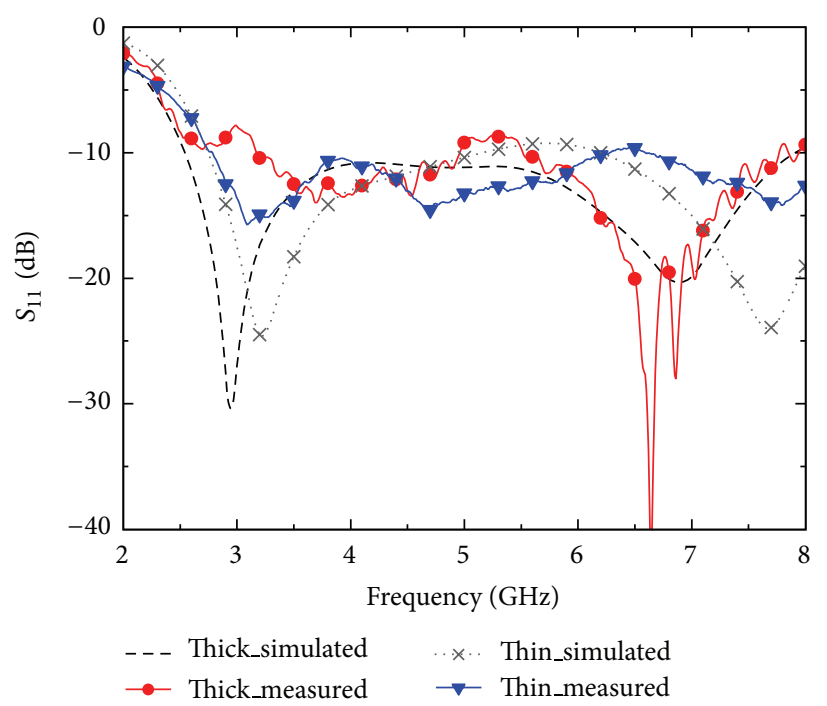

FIGURE 5: Simulated and measured return losses of the UWB antenna.

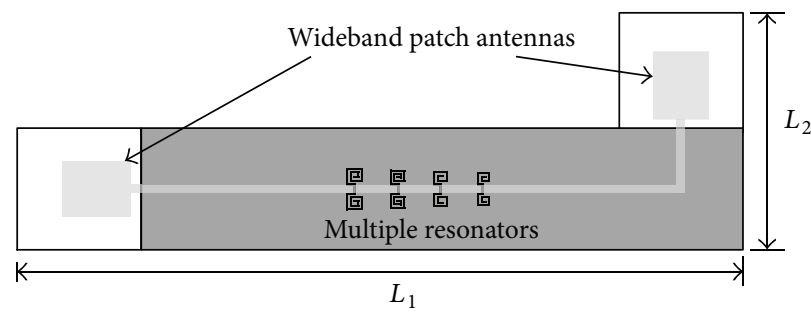

FIGURE 6: Layout of the microwave tag system (on the thin substrate).

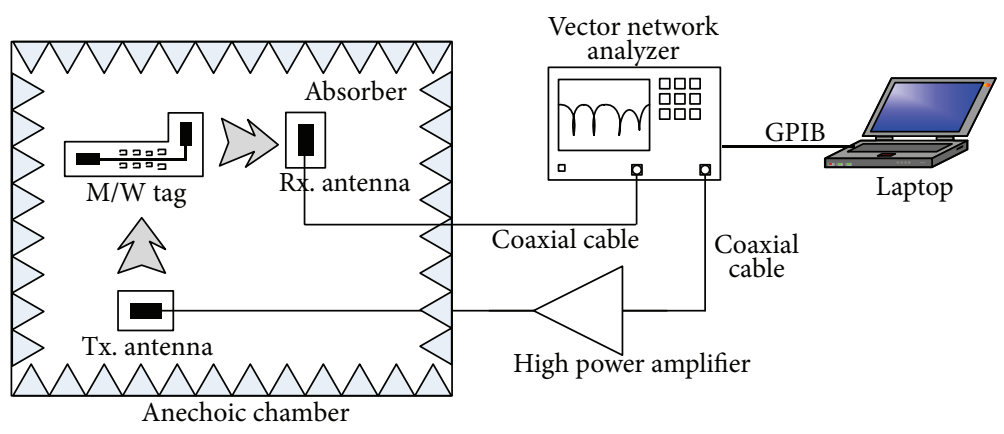

FIGURE 7: Interrogating system set up for wireless transmission tests.

\section{Implementation of the Microwave Tag System}

The proposed flexible microwave tag system with DGSs has been implemented on thick FR4 and thin flexible Teflon substrates. The tags with various 4 - or 5-bit codes are evaluated for the recognition of the ID characteristics. The resonator circuits and UWB antennas are integrated on a single PCB. Thanks to the full passive architecture, the proposed tag system can be implemented using a one-step printing fabrication process.
The layout of the implemented tag system is presented in Figure 6. In order to take the polarization diversity that can prevent jamming signals and cross talks, the transmitting and receiving antennas are mounted with an orthogonal orientation to each other. The full tag dimensions are $L_{1} \times$ $L_{2}=188.85 \times 72.75 \mathrm{~mm}^{2}$ for the thick substrate and $L_{1} \times L_{2}=$ $135.85 \times 63.25 \mathrm{~mm}^{2}$ for the thin substrate.

Figure 7 shows the interrogating system set up for wireless transmission tests. The performances of the wireless microwave tags have been evaluated in an anechoic chamber 
surrounded by electromagnetic absorbers to eliminate multipath effects. The microwave tag and Tx./Rx. reader antennas are fixed on stands fabricated from acrylic and styrofoam material. The Tx./Rx. reader antennas are connected to a Vector Network Analyzer (VNA; E5071C, Agilent Technologies Ltd.) by coaxial cables. For the purpose of providing sufficient transmitting power, a High Power Amplifier (HPA; ZVE-3W-83+, Mini-Circuits Ltd.) is inserted between the Tx. reader antenna and the VNA port. The HPA presents a high gain of $40 \mathrm{~dB}$ and a large dynamic range of $P_{1 \mathrm{~dB}}=$ $33 \mathrm{dBm}$ over $2-8 \mathrm{GHz}$. The received RF signal at the $\mathrm{Rx}$. antenna passes the multiple resonator circuit and reradiates via the Tx. antenna. The transmitted signal contains bit information of frequency resonance such as amplitude variations and phase nonlinearities, which are compared at the reader system. The detected information has been decoded and analyzed using a laptop computer connected to the VNA.

\section{Performance Evaluation of the Proposed Flexible Tag System}

In this section, the performance of the proposed tag system is evaluated from two experimental phases. While one experimental phase involves cognitive capability tests that correspond to the bent rate of the flexible thin tag, the other experimental phase is the ID code recognition test on a wireless transmission environment. Each ID code has been presented by measuring the relative insertion losses. The aggregator system organized by the VNA was calibrated to enhance the bit resolution. The calibration was performed by measuring a reference tag that has the same size and substrate as those of the devise under test (DUT) tag without any resonators. Because the reference tag represents the code of "00000," the codes of DUT can be more clearly detected by comparing the measured results between the DUT and the reference tag. The resonant ID codes have been recognized by computing the calibrated datum. In this measurement, the detection criteria are referred to the resolution of the $10 \mathrm{~dB}$ amplitude difference.

Flexibility tests have been conducted in order to consider the practical environments for which the flexible tag is applied. Because the tag should be operated in the state of wrinkled and bent shapes, performance variation can be created in a resonant frequency and radiation, and so forth. In this measurement, the proposed flexible tags were bent on a styrofoam jig with a specified radius of curvature, $r$, as shown in Figure 8. The flexible tags bent with various radiuses of curvature were measured in an anechoic chamber surrounded by electromagnetic absorbers.

Table 3 presents the measured relative insertion losses at the expected resonant frequencies from most significant bit (MSB) to least significant bit (LSB). The tag under the test is designed using the code "1101." Four conditions have been tested: an ideal flat surface and radiuses of curvature of $12 \mathrm{~mm}, 10 \mathrm{~mm}$, and $8 \mathrm{~mm}$, respectively. The ideal flat is considered for the reference values which present more than a $30 \mathrm{~dB}$ difference. As the radius of curvature decreases,
TABLE 3: Bit resolution for radiuses of curvature of flexible tags (code $1101)$.

\begin{tabular}{lcccc}
\hline$r$ & $1(\mathrm{MSB})$ & 1 & 0 & $1(\mathrm{LSB})$ \\
\hline Ideal & -47.62 & -40.41 & -7.73 & -41.51 \\
12 & -29.28 & -15.40 & -0.05 & -13.60 \\
10 & -18.70 & -22.99 & -1.96 & -11.01 \\
8 & -22.92 & -4.36 & -1.14 & -10.15 \\
\hline
\end{tabular}

the resolution becomes degraded. In the case of $r=8 \mathrm{~mm}$, the amplitude difference of code " 1 " and " 0 " is reduced to less than $10 \mathrm{~dB}$. Therefore, the flexible tag has a bending limitation to resolve the resonant frequencies and radiation characteristics in order to mount the applications on amorphous materials.

Figure 9 presents the experimental results of the proposed microwave tags using DGSs on the thick and thin flexible substrates. In Figures 9(a) and 9(b), the code detections of the microwave tags on thick substrates are presented for "10101" and "11001," respectively. In Figures 9(c) and 9(d), the bit representations at the reader are shown for the tag systems with codes of " 1101 " and " 1011 " on thin substrates, respectively. The experimental results have presented good agreement with the $\mathrm{S} 21$ values of the resonator circuit without antennas. Even though some frequency shifts at the bit position are found, these are determined to be detectable for code recognitions. In addition, each bit represented by a band notch is well recognized for wireless transmission tests. Figure 10 shows photographs of the proposed microwave tags fabricated on the thick FR4 and thin flexible Teflon substrates.

\section{Conclusions}

In this paper, the multiple resonator-based microwave tag system with DGSs has been proposed in flexible fabrication technology. The proposed architecture can avoid the direct reflection from a reader antenna and present an excellent resolution to realize the resonant bits. The resonant circuits have been implemented on the thick and thin flexible substrates. The cognitive capabilities have been experimented for various curvatures of flexible tags and wireless transmission performances. From the measured results, wireless transmission performances have presented good agreement with the ideal resonant bit characteristics. Additionally, the design limitation has been presented for a radius of curvature of flexible tags. Therefore, compared with a conventional UHF RFID system, the proposed microwave tag system can be an excellent candidate for a contactless ID tag system with low-cost and mass-productive merits. It can overcome the limitation of conventional RFIDs by fabricating a one-step process and mounting on amorphous items. 


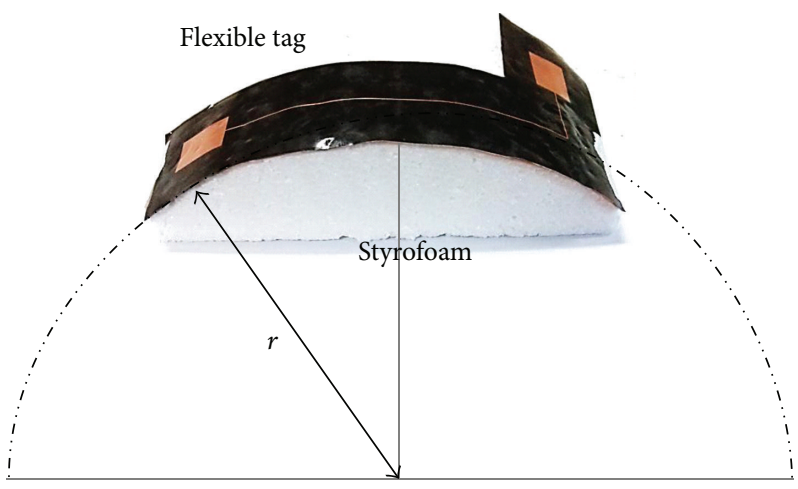

FIGURE 8: Photograph of the bent flexible tag mounted on a bending jig.

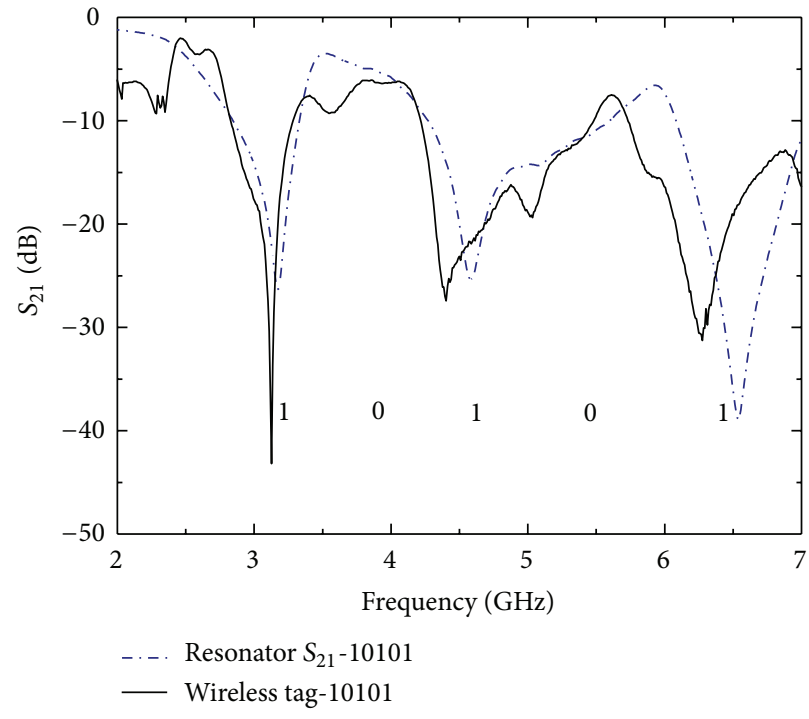

(a) Code 10101 on a thick substrate

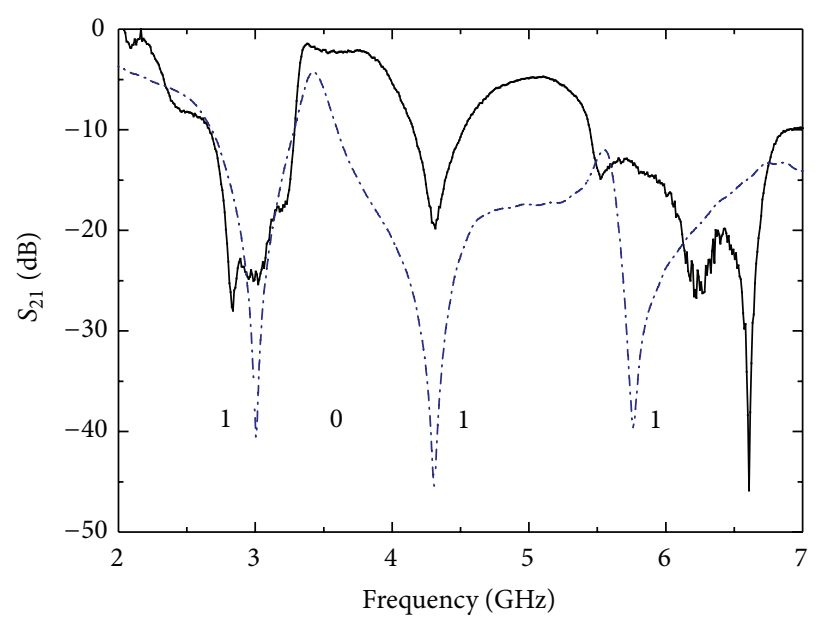

-... Resonator $S_{21}-1011$

- Wireless tag-1011

(c) Code 1011 on a thin substrate

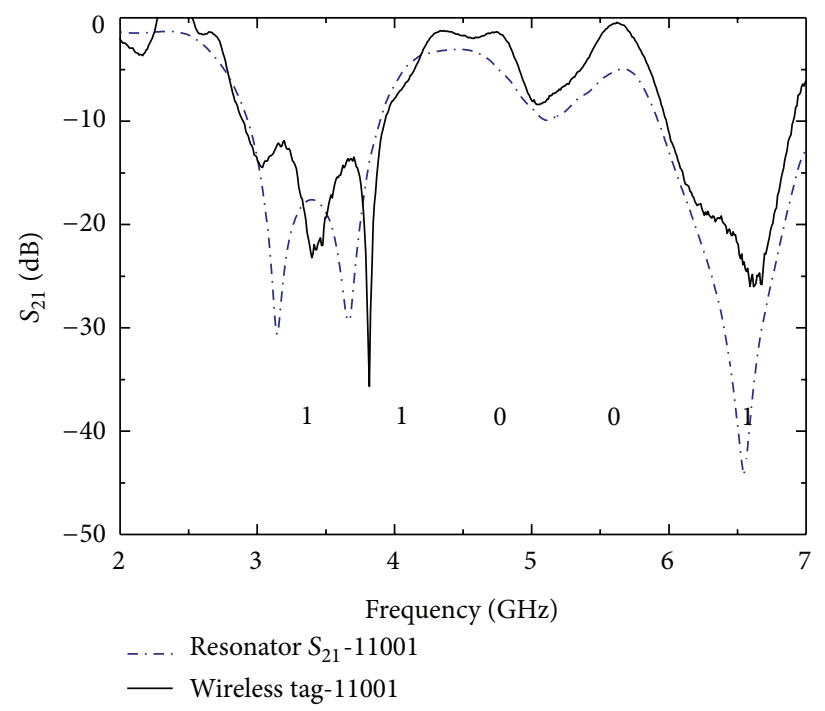

(b) Code 11001 on a thick substrate

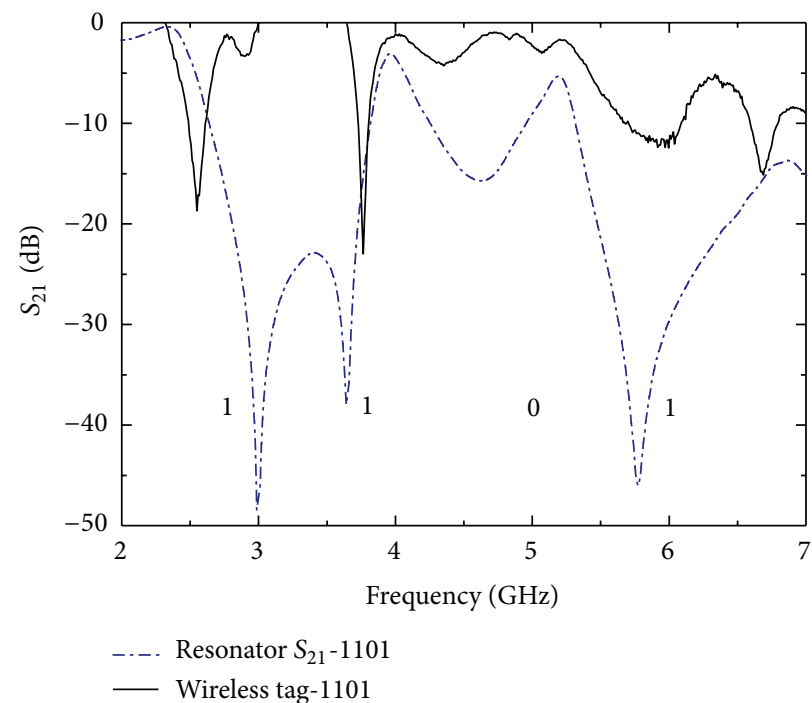

(d) Code 1101 on a thin substrate

FIGURE 9: Measured results of the proposed microwave tag in wireless experiments. 


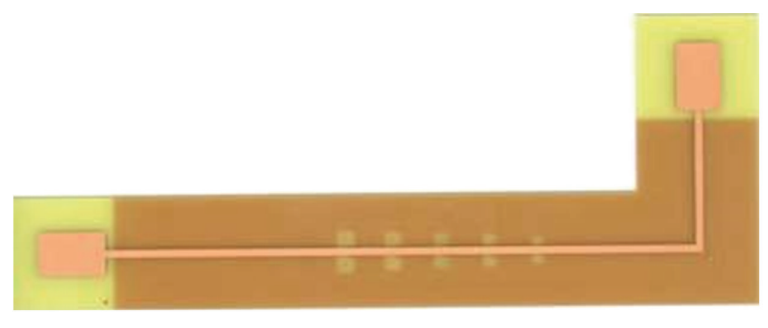

Front side

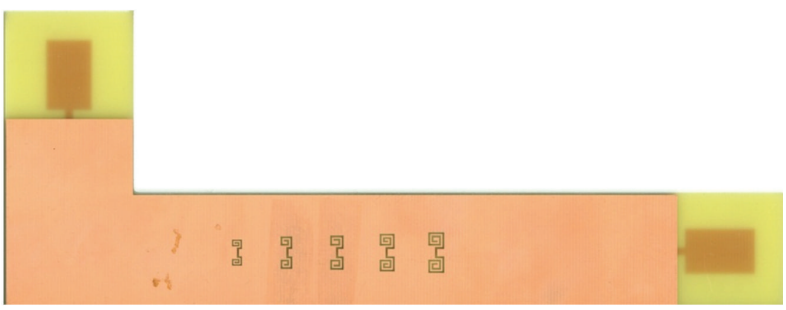

Rear side

(a)

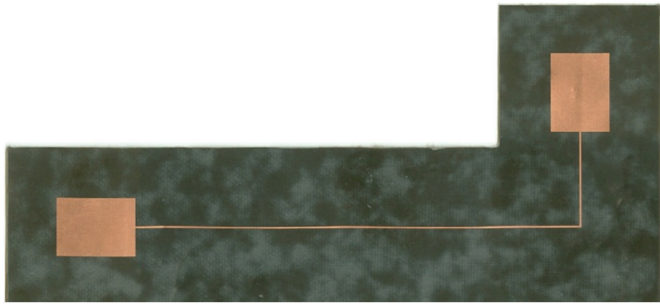

Front side

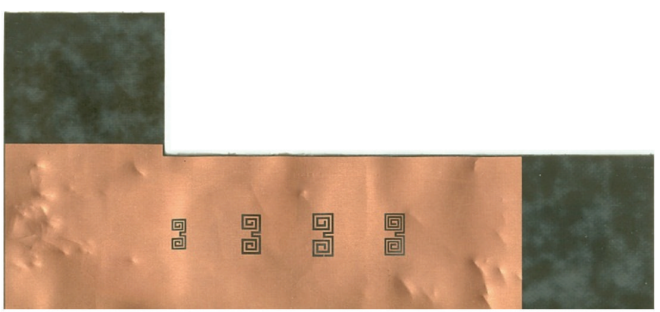

Rear side

(b)

FIGURE 10: Photographs of implemented microwave tag systems: (a) on the thick substrate, (b) on the flexible thin substrate.

\section{References}

[1] S. Preradovic and N. C. Karmakar, "Chipless RFID: bar code of the future," IEEE Microwave Magazine, vol. 11, no. 7, pp. 87-97, 2010.

[2] F. Michahelles, F. Thiesse, A. Schmidt, and J. R. Williams, "Pervasive RFID and near field communication technology," IEEE Pervasive Computing, vol. 6, no. 3, pp. 94-97, 2007.

[3] S.-M. Han, O. Popov, and A. Dmitriev, "Flexible chaotic UWB communication system with adjustable channel bandwidth in CMOS technology," IEEE Transactions on Microwave Theory and Techniques, vol. 56, no. 10, pp. 2229-2236, 2008.

[4] I. Jalaly and I. D. Robertson, "RF barcodes using multiple frequency bands," in Proceedings of the IEEE MTT-S International Microwave Symposium, pp. 139-142, June 2005.

[5] S. Härmä, V. P. Plessky, X. Li, and P. Hartogh, "Feasibility of ultra-wideband SAW RFID tags meeting FCC rules," IEEE Transactions on Ultrasonics, Ferroelectrics, and Frequency Control, vol. 56, no. 4, pp. 812-820, 2009.

[6] S. Preradovic, I. Balbin, N. C. Karmakar, and G. F. Swiegers, "Multiresonator-based chipless RFID system for low-cost item tracking," IEEE Transactions on Microwave Theory and Techniques, vol. 57, no. 5, pp. 1411-1419, 2009.

[7] M. Schüßler, C. Mandel, M. Maasch, A. Giere, and R. Jakoby, "Phase modulation scheme for chipless RFID- and wireless sensor tags," in Proceedings of the Asia Pacific Microwave Conference 2009 (APMC '09), pp. 229-232, Singapore, December 2009.

[8] S. Preradovic and N. C. Karmakar, "Multiresonator based chipless RFID tag and dedicated RFID reader," in Proceedings of the IEEE MTT-S International Microwave Symposium (MTT '10), pp. 1520-1523, May 2010.

[9] A. Islam, S. Bhuiyan, and N. Karmakar, "A novel compact chipless RFID tag and near-field reader," in Proceedings of the Asia Pacific Microwave Conference, pp. 1518-1521, December 2011.

[10] H.-S. Jang, W.-G. Lim, K.-S. Oh, S.-M. Moon, and J.-W. Yu, "Design of low-cost chipless system using printable chipless tag with electromagnetic code," IEEE Microwave and Wireless Components Letters, vol. 20, no. 11, pp. 640-642, 2010.

[11] J. Jung, H. Lee, and Y. Lim, "Broadband flexible comb-shaped monopole antenna," IET Microwaves, Antennas and Propagation, vol. 3, no. 2, pp. 325-332, 2009.

[12] S. Zhu and R. Langley, "Dual-band wearable textile antenna on an EBG substrate," IEEE Transactions on Antennas and Propagation, vol. 57, no. 4, pp. 926-935, 2009.

[13] C.-S. Kim, J. S. Lim, S. Nam, K. Y. Kang, and D. Ahn, "Equivalent circuit modeling of spiral defected ground structure for microstrip line," Electronics Letters, vol. 38, no. 19, pp. 1109-1110, 2002.

[14] D. Ahn, J. S. Park, C. S. Kim, J. Kim, Y. Qian, and T. Itoh, "A design of the low-pass filter using the novel microstrip defected ground structure," IEEE Transactions on Microwave Theory and Techniques, vol. 49, no. 1, pp. 86-93, 2001.

[15] T. Aboufoul, A. Alomainy, and C. Parini, "Reconfiguring UWB monopole antenna for cognitive radio applications using GaAs FET switches," IEEE Antennas and Wireless Propagation Letters, vol. 11, pp. 392-394, 2012.

[16] P. Moeikham, C. Mahatthanajatuphat, and P. Akkaraekthalin, "A compact UWB antenna with a quarter-wavelength strip in a rectangular slot for $5.5 \mathrm{GHz}$ band notch," International Journal of Antennas and Propagation, vol. 2013, no. 9, Article ID 574128, 2013.

[17] C. A. Balanis, Antenna Theory, Wiley \& Sons, New York, NY, USA, 2nd edition, 2001. 

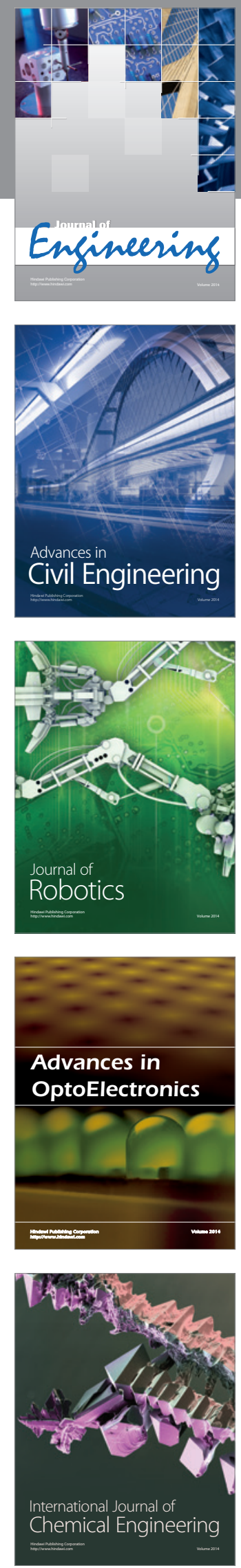

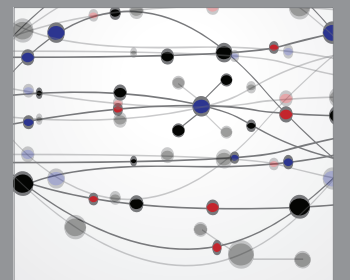

The Scientific World Journal
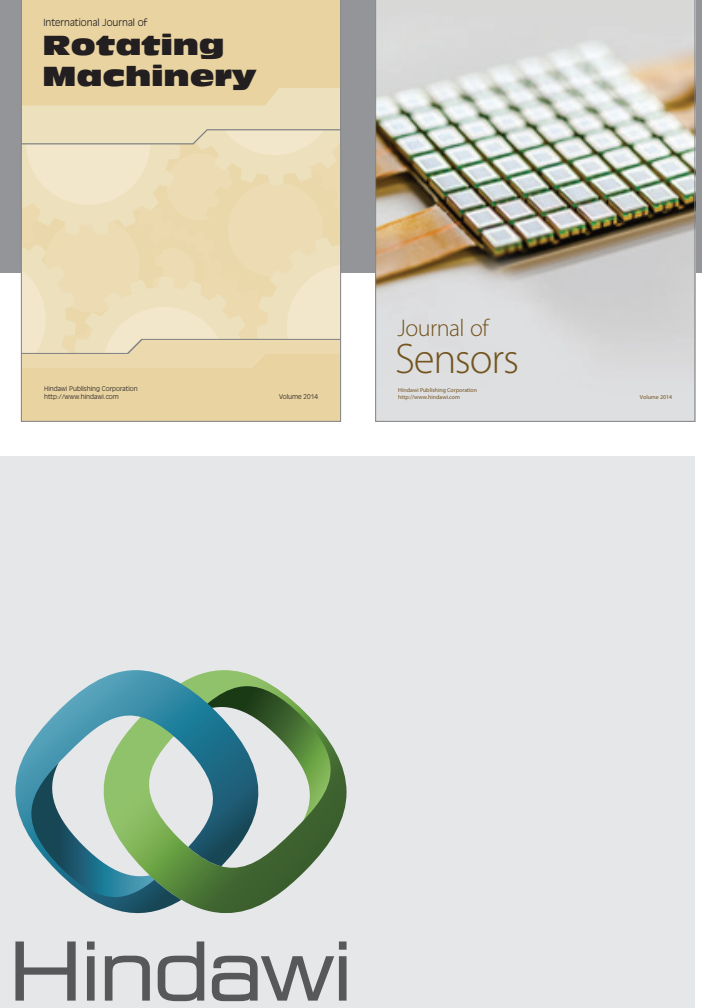

Submit your manuscripts at http://www.hindawi.com
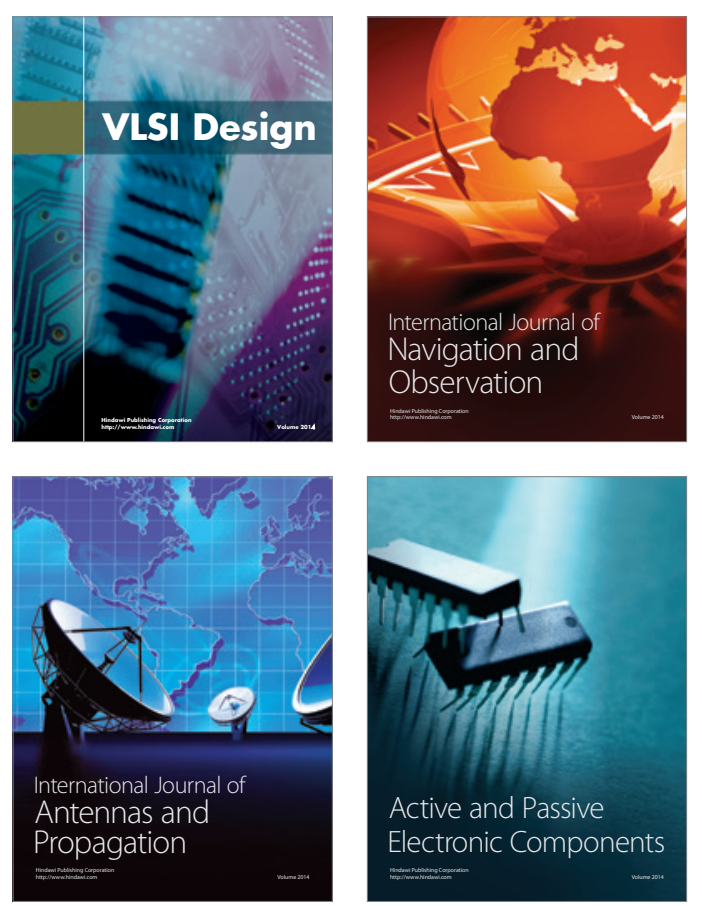
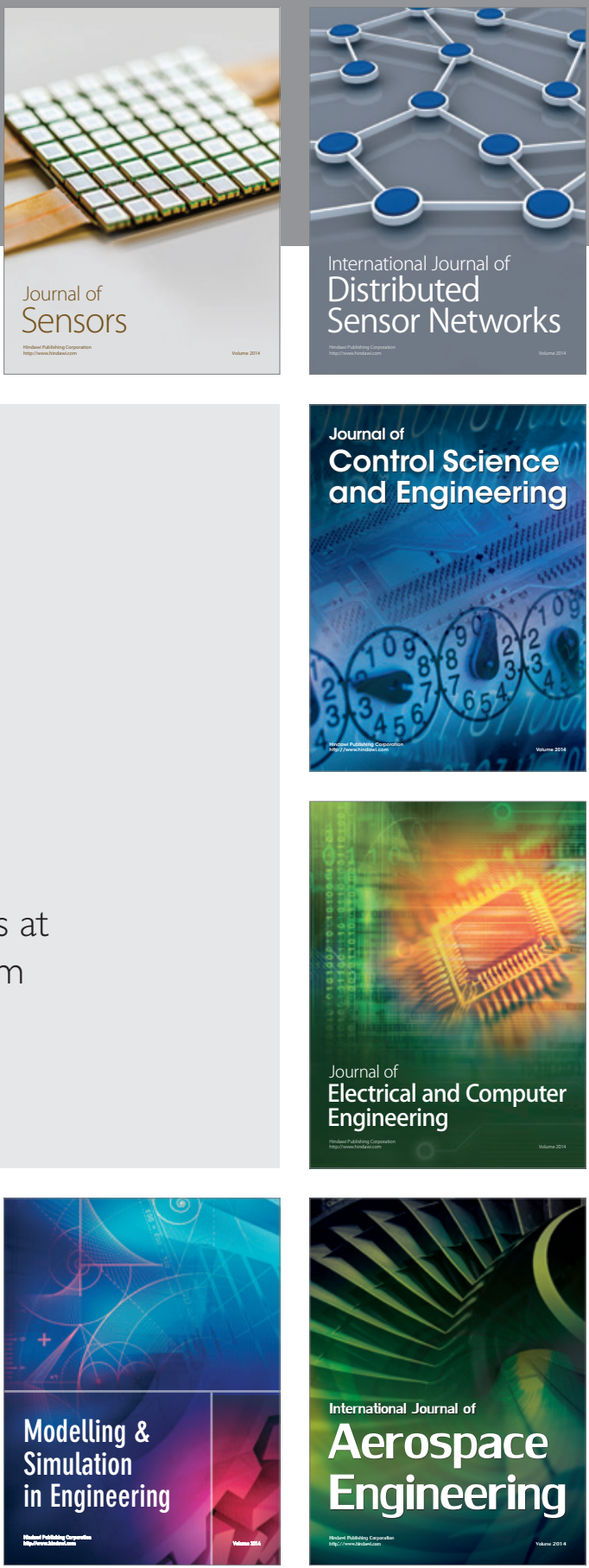

Journal of

Control Science

and Engineering
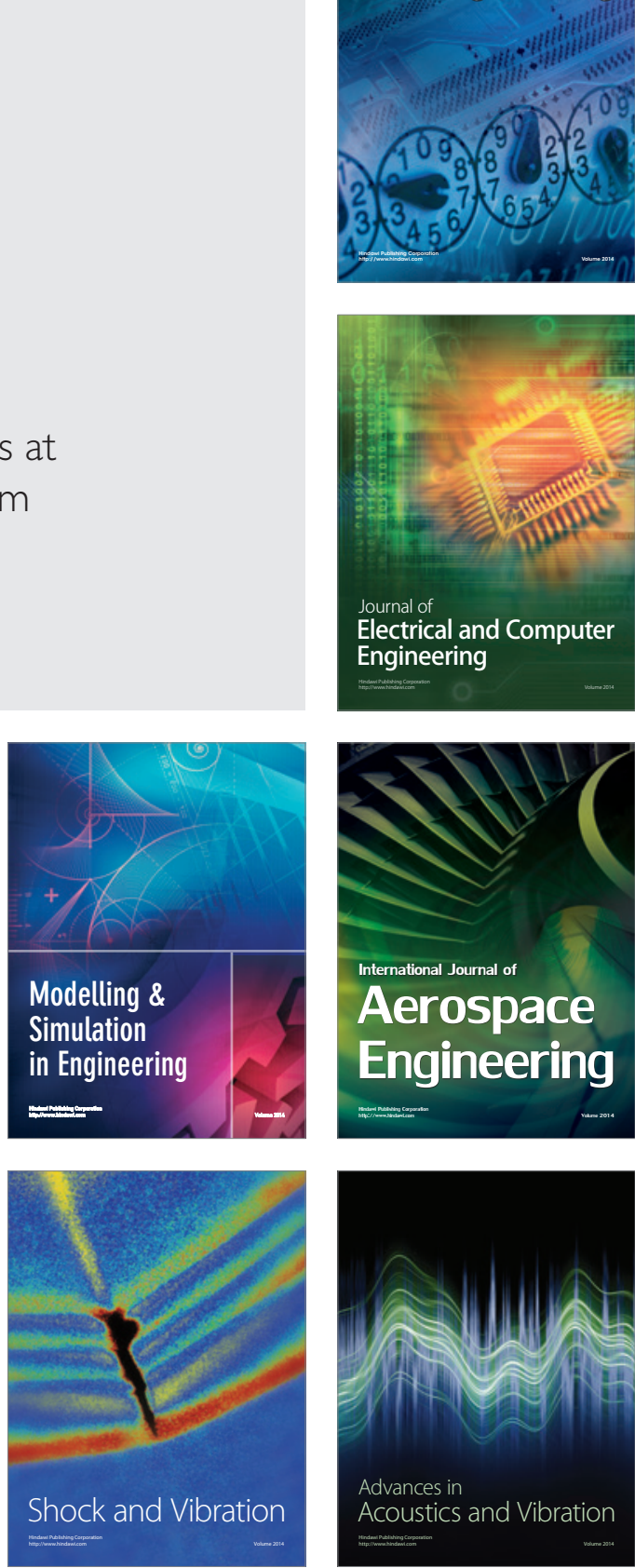